\title{
Research and Development of On-line Monitoring System for HV Overhead Transmission Lines
}

\author{
Zhilong Gao ${ }^{1,2, a}$, Dongtao Wang ${ }^{1,2, b}$ and Xiaocen Cai ${ }^{1,2, c}$ \\ ${ }^{1}$ Tianjin Key Laboratory of Information Sensing and Intelligent Control, Tianjin 300222, China \\ ${ }^{2}$ School of Automation and Electrical Engineering,Tianjin University of Technology and Education, \\ Tianjin 300222, China \\ agz190507@163.com, bdongtwang@sohu.com, ccxcandtoni@163.com
}

Keywords:high voltage; overhead transmission lines; monitoring system; wireless communication.

\begin{abstract}
As an important part of the power system, the running state of HV overhead transmission lines has direct influence on the security and reliability of power system. So it is important to develop an on-line monitoring system of HV overhead transmission lines. In this paper, a micro controller and wireless communication based high voltage transmission line monitoring system is developed. This system can monitor state of HV overhead transmission lines, such as micro-meteorology, icing, conductor wind deflection and galloping, tower inclination, tower leaning or collapse, conductor breaking, and insulator flashover. The software and hardware of monitoring system is introduced. Data given by the monitoring system can be used to analysis and predict the state of HV transmission lines and guide operators making maintenance plan.
\end{abstract}

\section{Introduction}

In recent years, with the implementation of projects for sending power from west to east, UHV and HV overhead transmission lines developed fast in China. Now China has almost the longest HV transmission lines in the world, and these lines is widely distributed in vast area along all country. As environment is complex and meteorology condition is bad, the place is difficult to access for maintenance staff in outfield. The monitoring for state of overhead lines is based on regular patrol of people on site. That consumed large amount of people and goods, and will enlarge the scope of the fault due to detect work delayed. So the design of a remote monitoring system of overhead lines state is very important for keeping power equipment in normal operation ${ }^{[1-2]}$.

As an important part of the power system, the running state of HV overhead transmission lines has direct influence on the security and reliability of power system ${ }^{[3-4]}$. According to statistical results of power industry, faults of $\mathrm{HV}$ overhead transmission lines caused by icing, conductor wind deflection and galloping, and insulator flashover frequently occurred and lead to large losses for enterprise and society. So it is important to develop an on-line monitoring system of HV overhead transmission lines ${ }^{[5-7]}$.

In this paper, a high voltage transmission line monitoring system based on micro controller and wireless communication is developed. This system can monitor state of HV overhead transmission lines, such as micro-meteorology, icing, conductor wind deflection and galloping, tower inclination, tower leaning or collapse, conductor breaking, and insulator flashover. The software and hardware of monitoring system is introduced.

\section{Hardware design of monitoring system}

\subsection{Design of System general plan}

The configuration of monitoring system is show as Fig.1. An hierarchical heterogeneous wireless sensor network of Zigbee is used in terminal units, and GPRS technology is used in remote data communication. The collected data is transferred to monitoring center by wireless network. 


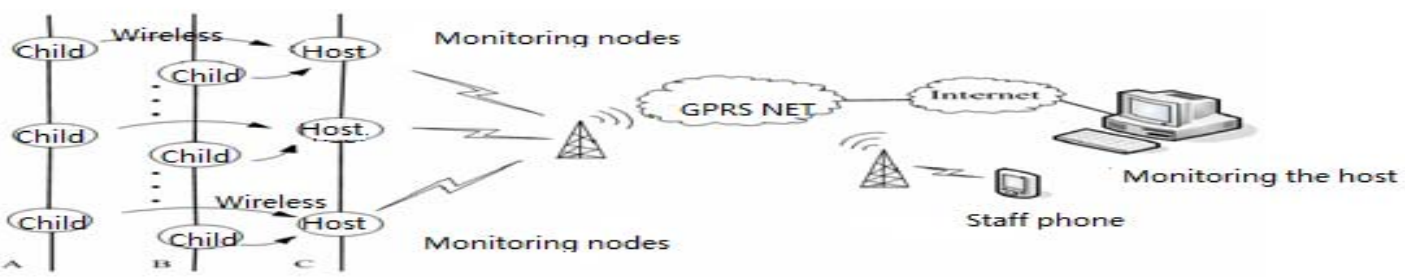

Fig.1 Monitoring system configuration

\subsection{Design of wireless sensor network of Zigbee}

ZigBee is a reliable, inter-operable, low power, low cost and easy-to-use wireless communication technology ${ }^{[8]}$. As an open wireless standard, it provides the foundation for the Internet of Things by enabling simple and smart objects to work together. ZigBee can be used in data communication for industry field automation. Low-power is another advantage of ZigBee. ZigBee allows devices to last for years on a single battery. These advantages of ZigBee can meet the needs of monitoring system well.

According to needs of monitoring points distribution of HV transmission lines, an hierarchical heterogeneous wireless sensor network is designed. The network consists of a backbone network and a sub-network, the latter adopting Zigbee technology being responsible for collecting images and scalar data.The backbone network is based on IEEE 802.11 multi-hop network that enables reliable long-distance transport.

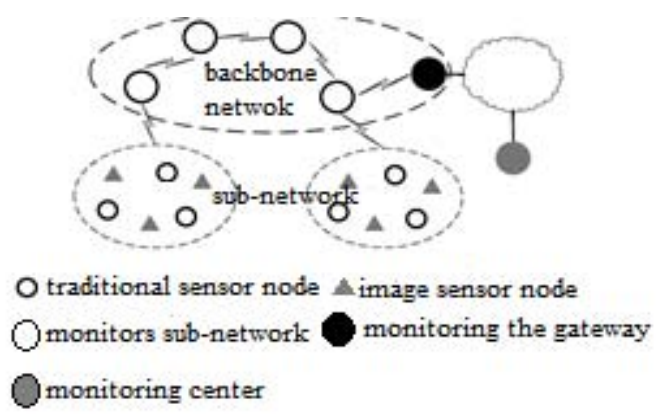

Fig. 2 Heterogeneous sensor network topology structure

\subsection{Design of system hardware}

\subsubsection{Monitoring substation}

Monitoring substation is consist of main board and sub-board. The MCU of main board is a low power single chip for communication. Its input voltage is $5 \mathrm{~V}$, and operation current is less than $3 \mathrm{~A}$.

1) The main board has $128 \mathrm{MB}$ SDRAM, 32MB Flash memory, a Wifi module with 2 miniPCI interface, ethernet interface, asynchronous communication interface and SPI interface.

2) The sub-board is Zigbee module. Sub-board is connected with main board through SPI interface. Sub-board surport IEEE 802.15.4 communication protocol and realizes protocol stack of Zigbee.

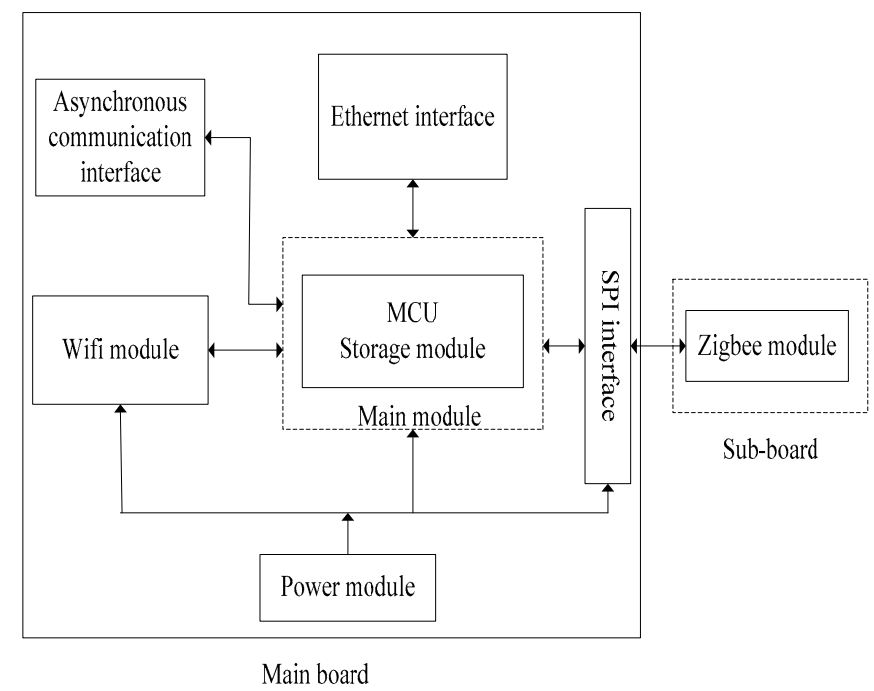

Fig.3 Monitoring substation 


\subsubsection{Sense node}

MC9S08GT60 is adopted for the micro control unit in sense node. MC9S08GT60 is an 8-bit low cost and low power MCU. It has Flash, on-chip RAM, 8-channel 10 bits ADC, two serial communication interfaces(SCI), and a serial peripheral interface(SPI).

MC13193 is used for RF Transceiver. MC13193 is a short range, low power, 2.4 GHz ISM band transceiver which contains a complete 802.15.4 PHY/MAC and the ZigBee protocol stack supporting star and mesh networking. The MC13193 provides a cost effective solution for short-range data links and networks.MC13193 is connected to MCU with serial peripheral interface(SPI). The configuration of sensor node is shown as Fig. 4.

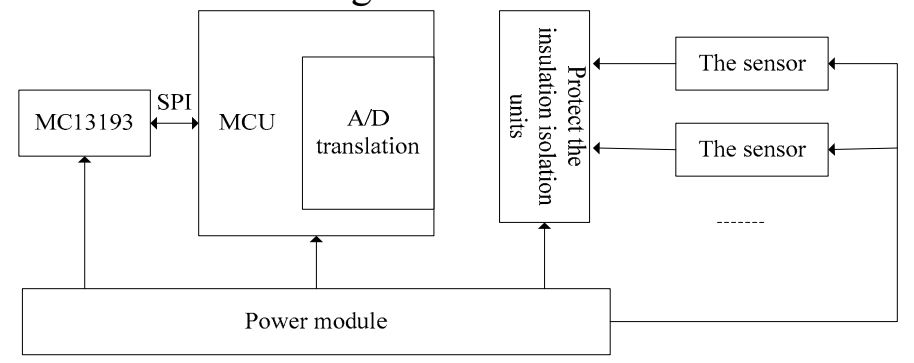

Fig.4 Sensor node configuration

\subsubsection{Lines temperature measurement}

DS18B20 is used for lines temperature measurement. DS18B20 is a temperature sensor with single line bus. DS18B20 is a micro chip with low power, high performance and stronger anti-interference ability. It can exchange temperature signal into digital signal, so it is very easy to connected with MCU.

\subsubsection{Environment temperature measurement}

An integrated temperature and humidity sensors SHT71 is selected for environment temperature measurement. SHT71 command codeSHT71 connects with micro controller through a two line serial interface.

\subsubsection{Power supply module}

Because of sensor nodes and monitoring substations are placed on lines in outside environment, the cell can not be replaced in time, so power supply is the key problem for monitoring system. The sensor node is low power equipment, its power can be supplied though electromagnetic induction coil with transmission lines. Electromagnetic induction coils are put on HV transmission lines. The output voltage of coils is transferred by steady voltage chip LM2576HV and AMS1117-3.3 into DC 3.3V. LM2576HV stable the voltage to $5.3 \mathrm{~V}$, then AMS1117-3.3 stable the output voltage to $3.3 \mathrm{~V}$. The maximum output current of AMS1117-3.3 is 1A, which can meet needs of sensor node. Regulating circuit principle diagram is shown as Fig 5.

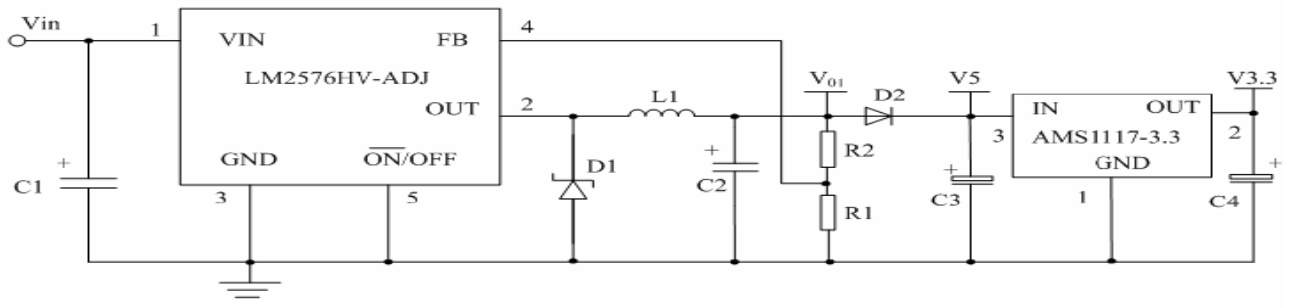

Fig.5 Regulating circuit principle diagram

\subsection{Design of system software}

The flowchart of of senor node is shown as Fig.6. The initialization of MCU is done first. Then select the communication channel, let MCU and MC13193 get into low-power state for preparing receiver. If a command is received and the command is legal, then make the MCU and MC13193 get out of low-power state, start the sensor and AD converter. When $\mathrm{AD}$ convert is end, then transfer data to monitoring substation. Else, if the command is not received or the command is illegal, then wait for next command. 


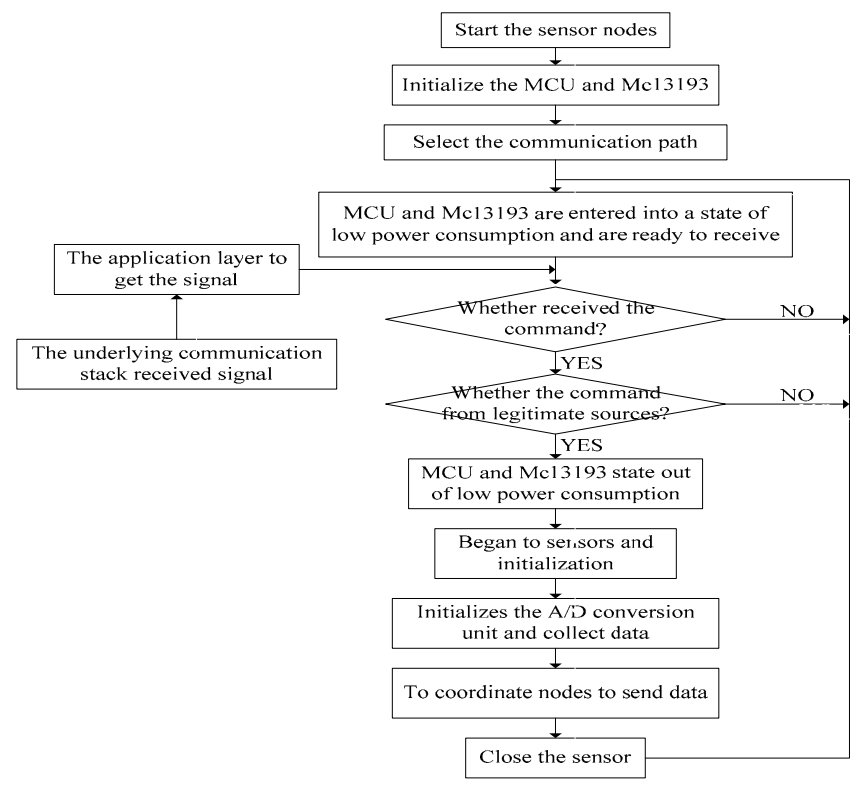

Fig.6 Flowchart of senor node

\section{Summary}

In this paper, a micro controller and wireless communication based high voltage transmission line monitoring system is developed. This system can monitor state of HV overhead transmission lines, such as micro-meteorology, icing, conductor wind deflection and galloping, tower inclination, tower leaning or collapse, conductor breaking, and insulator flashover. The software and hardware of monitoring system is introduced. This monitoring system can be used to analysis and predict the state of $\mathrm{HV}$ transmission lines and guide operators making maintenance plan.

\section{References}

[1] Liu Qi. On-line monitoring for high voltage transmission lines. Journal of business issue of science and technology, 2011, 24 (1) : 189-190.

[2] Fu Zheng-cai, Wu Bin, Huang Xian-dong, etal. On line monitoring system based on GSM network for transmission line fault. High Voltage Engineering, 2007,33（5）:69-72.

[3] Girgis A A,et al. A new fault location technique for two and three-terminal lines. IEE TransRWRD,1992,7(1):98-107.

[4] Qin Lijun Ma Jiyan etc. Intelligent distribution network and its key technology. Beijing: China power press, 2010.10.

[5] Huang Xin-bo, Sun Qindong, Cheng Ronggui, etal. Mechanical analysis on transmission line conductor ricing and application of on-line monitoring system. Automation of Electric Power Systems, 2007, 31(14):98-101.

[6] Wang Sheng-xue, Wu Guang-yu, Fan Jian-bin, etal. Study on flashover of suspension insulator string caused by windage yaw in $500 \mathrm{kV}$ transmissionlines. Power System Technology, 2008, 32(9): 65-69.

[7] Hang Xinbo, Liu Jiabing, Wang Xiangli, et al.On-line remote-monitoring system for transmission line insulator contamination based on the GPRS NET.Automation of Electric Power Systems, 2004, 28(21):92-96.

[8] Freescale Semiconductor, Inc. http://www.freescale.com. 